\title{
Acral Necrosis in a COVID-19-Infected Man Treated with Botulinum Toxin Type A
}

\author{
Mette Soelberg Schjødt Rikke Bech Anne Braae Olesen \\ Department of Dermatology, Aarhus University Hospital, Aarhus, Denmark
}

\section{Keywords}

COVID-19 · Acral necrosis $\cdot$ Botulinum toxin type A

\begin{abstract}
COVID-19 has been associated with acral ischemia and digital necrosis. Standard treatment of acral ischemia and digital or acral necrosis includes ongoing therapy with vasodilators and anticoagulants. However, these treatments are not always efficient to avoid the progression of necroses, which in the worst case can lead to amputation. Here, we report a case in which interdigital Botox ${ }^{\circledR}$ (botulinum toxin type A) nerve cord injection stopped the progression of acral necroses arising from an underlying vasculopathy due to COVID-19. Moreover, Botox ${ }^{\circledR}$ injection eliminated inflammation in the affected acral area within 2 weeks. This is the first case report to suggest Botox ${ }^{\circledR}$ injection as a new and improving treatment for acral necroses due to COVID-19.
\end{abstract}

\section{Introduction}

It is known that COVID-19-infected patients can develop coagulation abnormalities with an increased risk of thrombosis [1-3]. However, only a few studies have suggested that COVID-19 can mediate vasculopathy [2-4]. Vasculopathy represents any abnormality within a blood vessel and can affect any organ, including the skin [2]. This abnormality stems from either direct endothelial injury or is initiated by immune complexes affecting the endothelium [4]. Yet, COVID-19 has been associated with acral ischemia and digital necrosis. Treatment of acral ischemia includes vasodilators and anticoagulants to avoid the progression of necrosis. 
None of these treatments are very efficient in reducing the risk of auto or surgical amputation. Here, we report a case in which Botox ${ }^{\circledR}$ (botulinum toxin type A) injection stopped the progression of acral necroses due to COVID-19 infection.

\section{Case Report/Case Presentation}

A 75-year-old male hospitalized with COVID-19 and suffering from systemic sclerosis (SSc) of diffuse type, anti-RNA polymerase enzyme III positive, heart failure, atrial fibrillation, end-stage renal disease, and disseminated prostate cancer developed acral wet necroses on all fingers and toes and wet necroses on the heels/soles and in the sacral region on day 25 after confirmed COVID-19 infection. This was interpreted as an outcome of the combination of SSc and COVID-19 infection. The patient was treated with immunosuppressants and anticoagulants, among other medications - shown in Table 1. Botox ${ }^{\circledR}$ injection $100 \mathrm{U}$ was given to all fingers as interdigital nerve cord injection on phalanx II-V and in palma manus bilaterally, which remarkably dried out the necroses including the demarcation lines within 2 weeks, eliminated the inflammation, and stopped the progression of lesion extension (shown in Fig. 1). We observed that the Botox ${ }^{\circledR}$ injection stopped the inflammation demarcation line within a few days. Accordingly, the patient also experienced subjective pain relief within days after the treatment. Unfortunately, the patient died shortly after from severe heart and renal failure. Consent for publication was obtained from the next-of-kin, including publication of clinical photos. The method used in this case report was a review of the patient's medical record, including access to clinical photos and medication list.

\section{Discussion/Conclusion}

Botox ${ }^{\circledR}$ is used by dermatologists to treat peripheral vasculopathy such as Raynaud phenomenon and digital ulcers secondary to SSc by improving perfusion and relieving pain [5-7]. Botulinum toxin type A is produced by the bacterium Clostridium botulinum and expresses a periphery neuromuscular blocking effect. The toxin cleaves the SNARE proteins and indirectly inhibits neurotransmitter release of acetylcholine, but also glutamate, norepinephrine, substance $P$, and calcitonin gene-related peptide at the synapses and neuromuscular junction. Each neurotransmitter affects vascular tonus resulting in decreased vascular smooth muscle tone leading to vasodilatation [8]. The clinical effect of Botox ${ }^{\circledR}$ gradually occurs within 3-10 days and lasts approximately 12 weeks. We suggest that the braking of the inflammation demarcation line within a few days after Botox ${ }^{\circledR}$ injection results from neurotransmitter inhibition. The exact mechanism of decreased inflammation from Botox ${ }^{\circledR}$ is unknown. However, botulinum toxin inhibits the release of peripheral inflammatory mediators, including the pro-inflammatory calcitonin gene-related peptide, which give Botox ${ }^{\circledR}$ therapeutic effects in several neuroinflammatory disorders [9-13].

Our case developed extensive wet, acral necroses (finger, heels, and toes) and sacral necroses on day 25 after confirmed COVID-19 infection. The character of these lesions was much more comprehensive and aggressive than the normal progression of digital ulcers seen in SSc patients. Therefore, the lesions were interpreted as associated with the severe COVID-19 infection. We observed a notable effect on the fingertips' necroses, including the demarcation lines 2 weeks after Botox ${ }^{\circledR}$ injection - (shown in Fig. 1). We did not observe any improvement of the necroses on the patient's feet and sacral lesion. This may indicate that the patient benefitted from a one-time dose of interdigital injection with botulinum toxin type A.

\section{Karger'}


Case Reports

in Dermatology
Case Rep Dermatol 2021;13:568-573

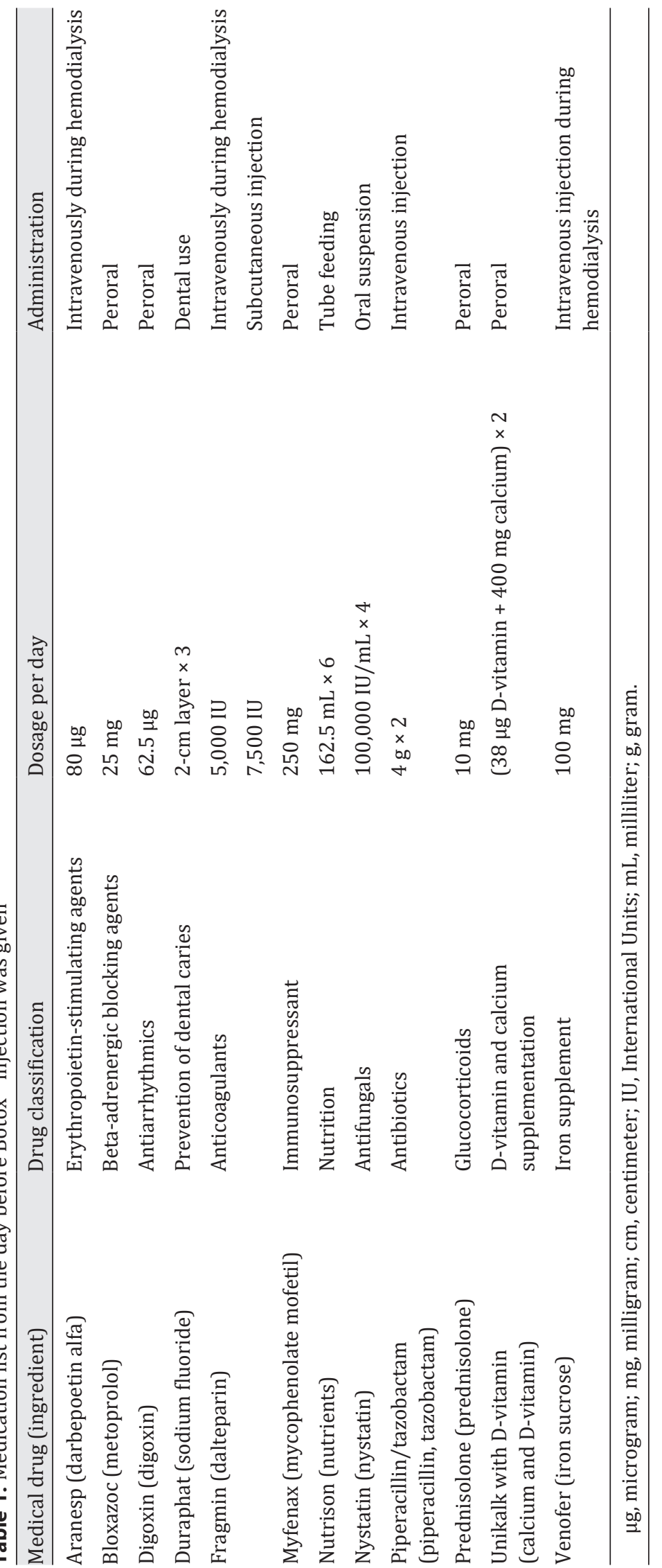

\section{Karger'}



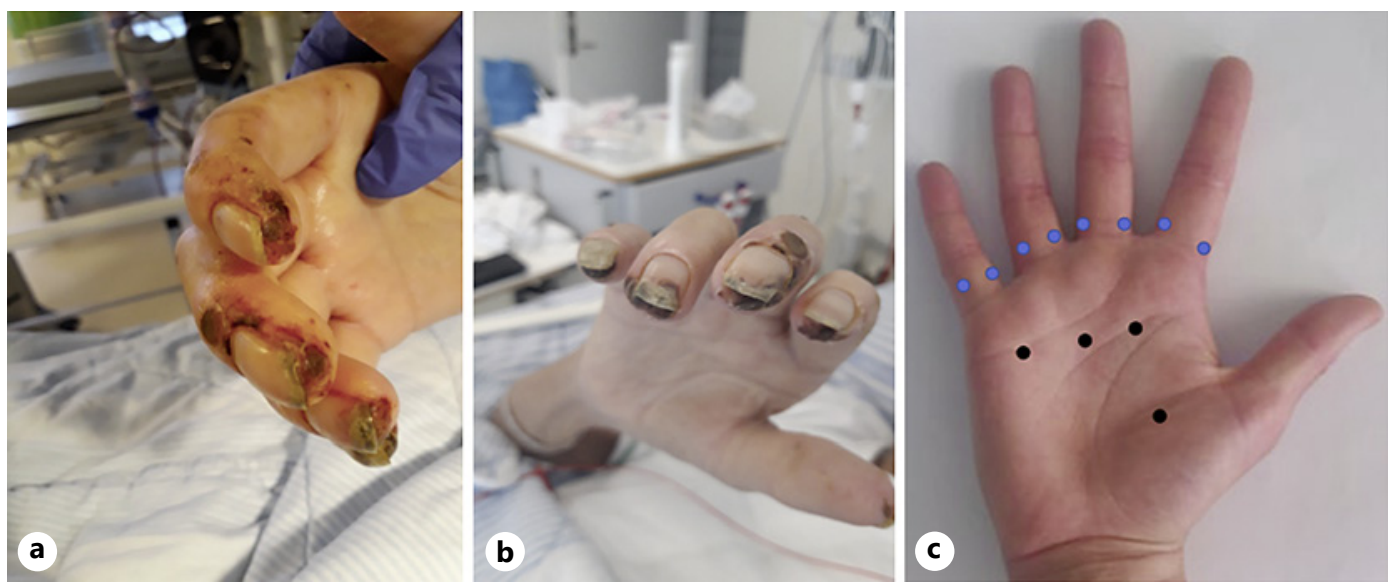

Fig. 1. a The day before Botox ${ }^{\circledR}$ injection: digits of the right hand showing wet necroses. $\mathbf{b}$ Two weeks after Botox ${ }^{\circledR}$ injection: dry digital necroses of the right hand with elimination of the inflammatory line of progression. No new necroses. c Distribution of Botox ${ }^{\circledR}$ injection $100 \mathrm{U}$ diluted in $2.2 \mathrm{~mL}$ saline solution in each hand. Blue dots, soft tissue injection at the level of the proximal phalanx base adjacent to digital arteries; black dots, soft tissue injection at the level of the superficial palmar arch.

Of course, we cannot rule out the possibility of other factors driving the observed improvement. For example, the interaction of all treatments and antibiotics combined with Botox ${ }^{\circledR}$ injection may have induced the improvement. However, our case had received peroral mycophenolate treatment for at least 3 years and prednisolone daily at least 6 months before the COVID-19 infection. In addition, antibiotic treatment was started 2 days before the Botox ${ }^{\circledR}$ treatment.

At the onset of acral necroses in our case, we had already seen improvement and halt of the necroses and demarcation lines after interdigital Botox ${ }^{\circledR}$ injections in another SSc patient who did develop extensive wet, acral necroses during COVID-19 infection. This other patient had active, progressive, wet acral necroses for 11 weeks before Botox ${ }^{\circledR}$ injection was given. However, the Botox ${ }^{\circledR}$ treatment stopped the wet progression lines within days. Before Botox ${ }^{\circledR}$ treatment, this patient was treated with extensive multiple therapies, which could not prevent the progressive wet necroses. The change from wet, progressive lines to dry demarcation lines was a compelling observation. Unfortunately, we are not allowed to give the full case story of this patient in this case report.

There is some evidence that COVID-19 mediates vasculopathy, and we consider that acral necroses due to COVID-19 arise from an underlying vasculopathy. We cannot with certainty rule out that the patient's underlying prostate cancer may have induced the severe wet necroses in our patient. However, it is not known that prostate cancer is associated with vasculopathy as opposed to COVID-19 infection.

The potential of botulinum toxin injection in the treatment of digital ischemia is investigated in a study among patients with posttraumatic hand injuries with convincing results. In the study, 5 cases and 6 controls with impending finger necrosis were treated with aspirin, protective dressings, and a warm milieu. In addition, cases were treated with Botox injection 80-100 U into the palm and adjacent to proximal arteries of affected fingers. The patients were precluded from treatment with intravenous thrombolytic agents because of polytrauma, concurrent medical illness, or pregnancy. Five controls went on to partial or complete digital amputation. In the Botox group, all digits were preserved. Moreover, cases experienced pain relief and improvement of digital perfusion [8]. The physiological mechanisms of Botox ${ }^{\circledR}$ are not fully elucidated. 
When treating patients with interdigital nerve cord injection with Botox ${ }^{\circledR}$, physicians should be aware of side effects such as injection site reactions, including pain, bleeding, bruising, redness, swelling or localized infection, and temporary digital paresthesia. Temporary digital paresthesia occurs in 1 out of 3 patients. Symptoms are most often present on day 3 after injection lasting up to 4 days. Severe but rare side effects include anaphylaxis, deep skin infection, and foreign body granuloma. Moreover, during injection, needle-related anxiety, or pain can cause vasovagal responses [14].

This is the first case report to suggest Botox ${ }^{\circledR}$ injection as a new and improving treatment for acral necroses due to COVID-19. Importantly, Botox ${ }^{\circledR}$ injection can easily be translated into clinical practice and requires a one-time dose instead of standard ongoing therapy [15]. Furthermore, from a patient perspective, Botox ${ }^{\circledR}$ injection preserves digits, relieves pain, improves the perfusion of fingers/toes, and maintains digital function. Thus, treatment with Botox ${ }^{\circledR}$ injection on acral necroses in COVID-19 patients in clinical practice could reduce medical costs by lowering the need for surgical amputation operations, thereby probably shortening hospital stay. We suggest that Botox ${ }^{\circledR}$ injection may decrease acral necrosis due to COVID-19-induced vasculopathy.

\section{Statement of Ethics}

This study protocol was reviewed and approved and signed by the Aarhus University Hospital, Chief Executive Officer Poul Blaabjerg, and the Head Consultant of the Department of Dermatology, Aarhus University Hospital Mette Deleuran, including approved access to the patient's record. Unfortunately, an approval reference number does not exist. Written informed consent was obtained from the next-of-kin for publication of the details of this medical case and any accompanying photos.

\section{Conflict of Interest Statement}

The authors have no conflicts of interest to declare.

\section{Funding Sources}

No funding to declare.

\section{Author Contributions}

A.B.O. and R.B. contributed to conceptualization; M.S.S. and A.B.O. contributed to methodology; M.S.S. contributed to writing - original draft preparation; R.B. and A.B.O. contributed to writing - review and editing; M.S.S., R.B., and A.B.O. contributed to visualization; R.B. and A.B.O. contributed to supervision. All authors have read and agreed to the published version of the manuscript.

\section{Data Availability Statement}

No data are available.

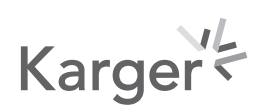




\section{References}

1 Lodigiani C, Iapichino G, Carenzo L, Cecconi M, Ferrazzi P, Sebastian T, et al. Venous and arterial thromboembolic complications in COVID-19 patients admitted to an academic hospital in Milan, Italy. Thromb Res. 2020; 191:9-14.

2 Labò N, Ohnuki H, Tosato G. Vasculopathy and coagulopathy associated with SARS-CoV-2 infection. Cells. 2020;9(7):1583.

3 Mondal R, Lahiri D, Deb S, Bandyopadhyay D, Shome G, Sarkar S, et al. COVID-19: are we dealing with a multisystem vasculopathy in disguise of a viral infection? J Thromb Thrombolysis. 2020;50(3):567-79.

4 Becker RC. COVID-19-associated vasculitis and vasculopathy. J Thromb Thrombolysis. 2020;50(3):499-511.

5 Bello RJ, Cooney CM, Melamed E, Follmar K, Yenokyan G, Leatherman G, et al. The therapeutic efficacy of botulinum toxin in treating scleroderma-associated Raynaud's phenomenon: a randomized, double-blind, placebocontrolled clinical trial. Arthritis Rheumatol. 2017;69(8):1661-9.

6 Segreto F, Marangi GF, Cerbone V, Persichetti P. The role of botulinum toxin A in the treatment of Raynaud phenomenon. Ann Plast Surg. 2016;77(3):318-23.

7 Guerra MG, Fonseca DGD, Samões B, Videira T, Pinto P. Is botulinum toxin useful in systemic sclerosis related peripheral vasculopathy? A literature review. Reumatol Clin. 2021 Jun-Jul;17(6):357-63.

8 Laarakker AS, Borah G. Botulinum toxin A salvage of ischemic hand trauma. Plast Reconstr Surg. 2020;145(1): 161-4.

9 Meng J, Ovsepian SV, Wang J, Pickering M, Sasse A, Aoki KR, et al. Activation of TRPV1 mediates calcitonin gene-related peptide release, which excites trigeminal sensory neurons and is attenuated by a retargeted botulinum toxin with anti-nociceptive potential. J Neurosci. 2009;29(15):4981-92.

10 Casale R, Tugnoli V. Botulinum toxin for pain. Drugs R D. 2008;9(1):11-27.

11 Pirazzini M, Rossetto 0, Eleopra R, Montecucco C. Botulinum neurotoxins: biology, pharmacology, and toxicology. Pharmacol Rev. 2017;69(2):200-35.

12 Borodic GE, Acquadro M, Johnson EA. Botulinum toxin therapy for pain and inflammatory disorders: mechanisms and therapeutic effects. Expert Opin Investig Drugs. 2001;10(8):1531-44.

13 Borodic GE, Caruso P, Acquadro M, Chick S. Parry-Romberg syndrome vasculopathy and its treatment with botulinum toxin. Ophthalmic Plast Reconstr Surg. 2014;30(1):e22-5.

14 Dashtipour K, Pedouim F. Botulinum toxin: preparations for clinical use, immunogenicity, side effects, and safety profile. Semin Neurol. 2016;36(1):29-33.

15 Laarakker ASS, Borah G. Reply: botulinum toxin A salvage of ischemic hand trauma. Plast Reconstr Surg. 2021; 147(4):704e. 